\title{
Malignant melanoma with liver and spleen metastases: case report
}

Gastroenterology Division, M edicine D epartment, U niversidade Federal de São Paulo/E scola Paulista de M edicina, São Paulo, Brazil

\section{abstract}

CONTEXT: The diagnosis of primary melanoma is easily confirmed after histological analysis of the lesion, whereas it is rarely diagnosed when the patient even has distant metastases.

DESIGN : Case report

CASE REPORT: M a lignant melanoma is responsible for about $1 \%$ of all deaths caused by cancer in the USA and only $3 \%$ of all malignant skin diseases. M alignant melanoma is a rare disease, although it corresponds to $65 \%$ of all deaths caused by skin cancer. The liver and spleen are rarely the first sites of melanoma metastases. This paper reports on the clinical picture of a patient with fatal malignant melanoma and hepatic and spleen metastases. As this was an unusual presentation, the melanoma diagnosis could only be made after pathological analysis of the skin and hepatic lesions.

KEY WORDS: M elanoma. Liver metastases. Spleen metastases.

\section{INTRODUCTION}

The diagnosis of primary melanoma is easily confirmed after histological analysis of the lesion, whereas it is rarely diagnosed when the patient even has distant metastases ( $8 \%$ of cases). ${ }^{1}$ This is probably due to the fact that the time between diagnosis of the primary lesion and the appearance of metastases is very long. Klaase et al. (1990) found an average time of 3 years for melanoma and metastases, among 30 patients studied. ${ }^{2}$

Malignant melanoma represents about $1 \%$ of all cancers and deaths in the USA but only $3 \%$ of all malignant skin diseases, although it is responsible for $65 \%$ of deaths caused by skin cancer. It shows a progressive increase in incidence with age, with the patients being mainly between 30 and 60 years old. Melanoma is more common among the white races. The skin pigment has a protective function in colored people, especially the black and yellow races. The most important cause of this cancer seems to be solar exposure. ${ }^{3}$ The primary lesions are located in: limbs (22\%), trunk (40\%), head and neck (15\%), and $16 \%$ in unknown sites (4). The most common sites of metastases found in the autopsy are: skin and subcutaneous tissue (75\%), lung (70\%), liver (68\%), small intestine (58\%), pancreas (53\%), heart (49\%), brain (39\%) and spleen (36\%). ${ }^{5}$ The average survival period for patients with non-visceral metastases is 7.2 months, but it falls to 2.4 months when liver metastases are considered, whether associated with other organs or not. ${ }^{1}$

\section{CASE REPORT}

A 60-year-old white Brazilian male was hospi- 
talized for investigation with a two month history of abdominal pain, altered intestinal function, lack of appetite and asthenia, accompanied by chills and night fever. The patient reported an unquantifiable weight loss and he had been smoking twenty cigarettes a day for the last 30 years.

Physical examination revealed that he had a regular general state, and he was anemic but not jaundiced or febrile. Nodular lesions were observed over the whole body of approximately $1 \mathrm{~cm}$ diameter, fiberelastic in nature, without infiltration into deeper tissues, covered only by skin of normal aspect. There was one nodule of approximately $2 \mathrm{~cm}$ diameter, in the posterior face of the left outer ear that was hardened, ulcerated and associated with nearby angiomas. The liver was observed to be $12 \mathrm{~cm}$ below the right costal margin, hardened on its costal edge, nodular, painful, and the Traube space was massive.

Pertinent laboratory findings are shown in Table 1. Biochemical assays showed normal bilirubin, aspartate aminotransaminase (AST) greater than alanine aminotransaminase (ALT), low serum albumin, altered prothrombin activity (PA), high cholestatic enzymes (alkaline phosphatase, AP; and gamma glutamyl transferase, gGT), and very high lactic dehydrogenase (LDH).

Oral endoscopy revealed 3 thin varicose cords and scarring from a duodenal ulcer. Sonography of the abdomen showed heterogeneous hepatomegaly and splenomegaly with multiple images suggestive of nodular metastatic lesions in the liver and spleen. There was a nodular image suggestive of peripancreatic ganglia and a left kidney cyst. Computed tomography of the abdomen confirmed the presence of hepatic and spleen nodules, suggestive of metastatic neoplasm (Figure 1). Colonoscopy showed hypertonic diverticular disease. Other exams such as bone scintigraphy with ${ }^{99 m}$ Tc-methylene diphosphonate (99mTc-MDP) showed heterogeneous distribution of the radioindicator in the ribs, and computed tomography of the thorax and bronchoscopy did not suggest any malignant lesions. Biopsies (skin and liver) revealed malignant melanoma in the lesion of the left outer ear and melanotic metastases in the subcutaneous tissues and hepatic nodules (Figure 2).

The patient's general state declined rapidly with weight loss, asthenia, painful abdominal distention radiating to the back, ascites, pleural spillage to the right, bilateral edema involving the lower extremities, dyspnea and torpor. After 22 days in hospital, he developed acute anemia and urinary hemorrhage. The final laboratory examinations are shown in Table 1. At this time the patient developed persistent acidosis $(\mathrm{pH}$ $=7.25$, bicarbonate $=12.1 \mathrm{mmol} / \mathrm{L}$ and $\mathrm{BE}=-12.8$ ) which did not respond to the treatment. Death occurred on the $24^{\text {th }}$ day.

\section{DISCUSSION}

The initial clinical diagnosis was of colorectal cancer with hepatic and splenic metastases, because of the changes in intestinal function and the fact that the large intestine is an usual primary source of liver metastases. ${ }^{6}$ The primary cancer situated on the left outer ear did not have the typical macroscopic characteristics of malignant melanoma. Therefore, the final diagnosis was only conclusive after histological analysis of the lesion.

Despite the low frequency of metastatic tumors in the spleen (4\%), these are more frequently encountered in autopsies of patients with melanoma (36\%). ${ }^{5}$ The diagnosis of metastatic melanoma in the spleen is rare, although this patient presented splenic metastases at the time of diagnosis. Marked splenomegaly is not common, probably because the average size of the nodules is $1.5 \mathrm{~cm} .^{5}$

The frequency with which the liver is observed to be the initial site of melanoma metastases is approximately $4 \%$. The initial clinical presentation includes weakness, anorexia, hepatomegaly and abnor-

\section{Table 1 - Results of blood examinations at the initial and in the terminal phases of the patient with malignant melanoma}

\begin{tabular}{lcc}
\hline Examinations & Entrance & Terminal phase \\
\hline $\mathrm{Hb} / \mathrm{Htc}$ & $11.2 / 33$ & $6.4 / 19$ \\
W hite blood cells & 12200 & 15500 \\
Differential (leukocytes) & $2 / 70 / 3 / 13 / 11$ & $21 / 64 / 0 / 9 / 6$ \\
Platelets & 336000 & 22700 \\
$\mathrm{AST} / \mathrm{ALT}$ & $55 / 47$ & $3152 / 554$ \\
$\mathrm{AP} / \mathrm{gGT}$ & $1156 / 477$ & $1518 / 348$ \\
$\mathrm{~TB} / \mathrm{CB}$ & $0.9 / 0.4$ & $1.1 / 0.8$ \\
$\mathrm{PA}$ & $57.7 \%$ & $44.7 \%$ \\
$\mathrm{Albumin}$ & 2.5 & 2.1 \\
$\mathrm{DH}$ & 5724 & 12765 \\
$\mathrm{~N}$ a/ K & $136 / 3.9$ & $128 / 6.0$ \\
$\mathrm{Hematuria}$ & $\mathrm{N}$ egative & 320,000 \\
$\mathrm{CEA}$ & $\mathrm{N}$ ormal & \\
AFP & $\mathrm{N}$ ormal & \\
HbSAg & N egative & \\
HCV antibody & N egative & \\
\hline
\end{tabular}

$\mathrm{Hb}$ - hemoglobin ( $\mathrm{g} / \mathrm{dl}$ ); $\mathrm{Htc}$ - hema tocrit (\%); Differential (metamyelocyte, segmented neutrophil, eosinophil, lymphocyte, monocyte); AST- aspartate a minotransa minase (nl: $<37 \mathrm{U} / \mathrm{L}$ ) and A LT-alanine a minotransa minase $(\mathrm{nl}:<40 \mathrm{U} / \mathrm{L}) ;$ AP- alkaline phosphatase (nl: 50-250 U/L) and gGTgamma glutamyl transferase (nl: 11-43 U/ L); TB- total bilirubin and CBconjugated bilirubin; PA - prothrombin activity; $\mathrm{LDH}$-lactic dehydrogenase; $\mathrm{N}$ a-sodium and K-potassium; Hematuria- red blood cells/ $\mathrm{ml}$ of urine; CEA-carcinoembryonic antigen; AFP-alpha fetus protein; $\mathrm{HbSAg-hepa-}$ titis $B$ surface antigen. 
mal liver function tests, ${ }^{5}$ which are common in any chronic hepatic disease. The initial laboratory tests (Table 1) showed our case to be chronic hepatic disease, with a cholestasis pattern. There was a significant increase in LDH, suggesting tissue necrosis, which could be described as tumoral invasion.

The initial investigation of abdominal tumor metastases, as for melanoma, should be done using sonography. Computed tomography and nuclear magnetic resonance should also be considered. Melanotic liver metastases are poorly vascularized in hepatic arteriography and small lesions may be easily missed. Diagnostic explorations via radioscintigraphy, especially using ${ }^{99 m}$ Tc-DMP, produce both false negative and false positive results and are thus not useful in the

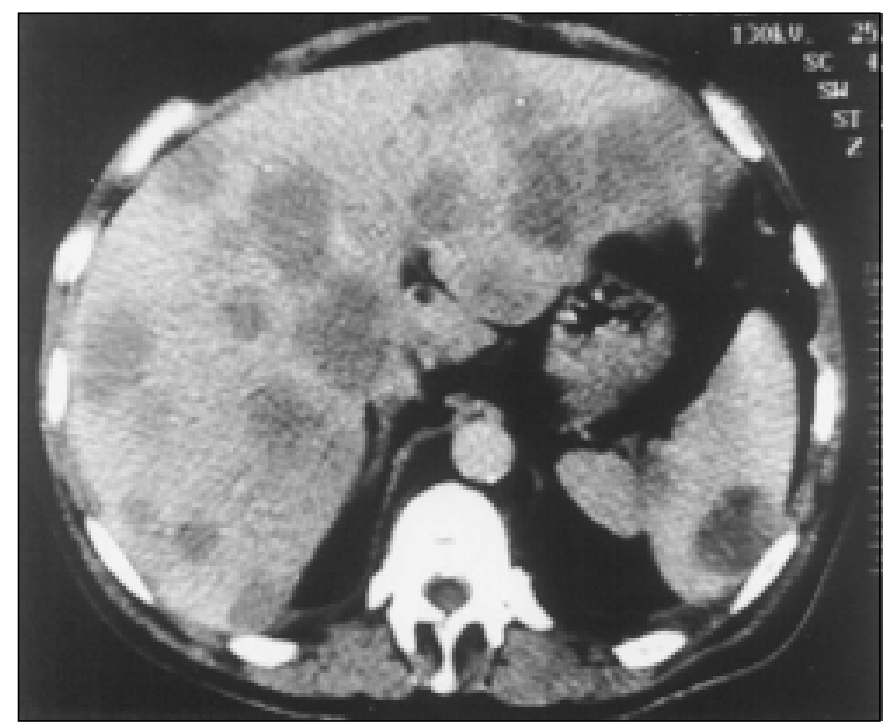

Figure 1 - Abdominal computed tomography, showing widespread hypodense nodules in the hepatic and spleen beds, corresponding to a metastatic neoplasm.

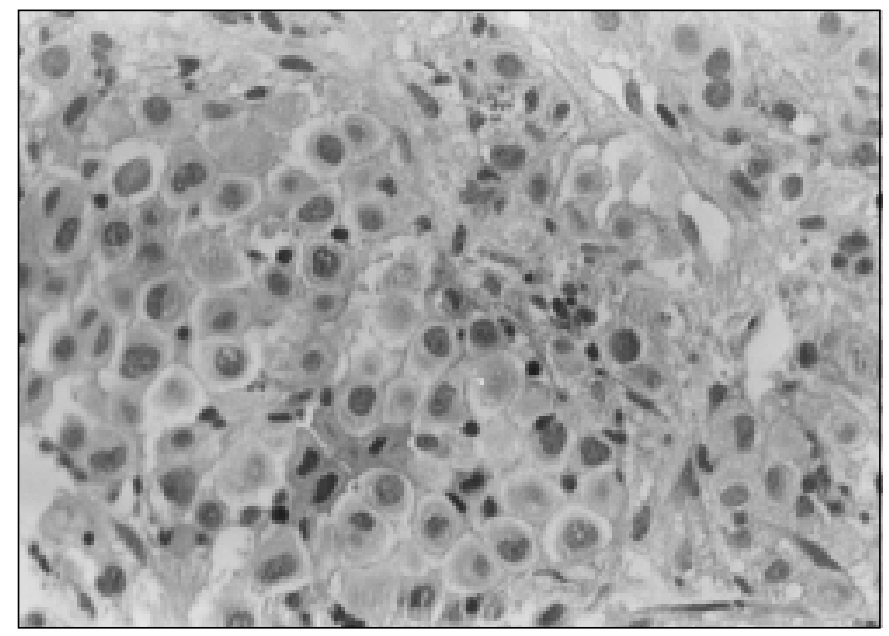

Figure 2 - High magnification photomicrograph showing metastatic melanoma in liver tissue. Large cells with vesicular nuclei, prominent nucleoli and eosinophilic cytoplasm (hematoxylin and eosin, 400X). initial phases of melanoma when there is no hepatic lesion. ${ }^{7}$ Although the liver biochemical tests showed hepatic alterations, the use of ${ }^{99 \mathrm{mTC}} \mathrm{TMMP}$ did not appear to add any other conclusion. Hepatic biopsy is a sensitive examination for the diagnosis of metastatic melanoma and the chances of a positive result are increased if the biopsy needle is directed into the lesion under ultrasound, computed tomography or peritoneoscopic guidance. Das Gupta and Brasfield (1964) showed that the incidence of hepatic metastases at necropsy is $68 \%$ and that the nodules are usually multiple, varying in size from 0.5 to $6.0 \mathrm{~cm}$ diameter, provoking hepatomegaly. ${ }^{5}$

Metastatic melanoma in the skin and/or subcutaneous tissue of the trunk and extremities is found in $75 \%$ of patients at autopsy. These metastases result from either lymphatic or vascular dissemination. Melanotic bone metastases are rare (2\%) and difficult to determine. ${ }^{5}$

The factors responsible for the death of our patient were probably hemorrhagic and metabolic complications. The destruction of the hepatic parenchyma by the tumor led to significant enzymatic alterations (Table 1), characterized by the increase of AST, ALT and $L D H$, the cholestatic pattern with high level of gGT and AP, and low serum level of albumin and altered PA. Jaundice usually indicates advanced hepatic disease, ${ }^{5}$ which did not occur in our patient. However the pattern of chronic hepatic disease also contributed to the death of our patient.

At the terminal phase, metabolic acidosis was observed. This could be a consequence of increased production of lactic acid due to inadequate peripheral tissue oxygenation or al tered metabolic functions such as insufficient capacity for hepatic clearance of lactate caused by the replacement of normal liver mass by tumor. It is suspected that these metastatic lesions cause local hypermetabolism, thereby worsening hepatic hypoxia and exacerbating lactic acidosis. ${ }^{8}$ Furthermore, the development of ascites and gradual edema of the lower extremities suggests retroperitoneal or mesenteric metastases, which usually have a very bad prognosis. ${ }^{5}$ The patient developed very pronounced anemia due to hematuria exacerbated by low platelets and altered PA. Another contribution to this fatal evolution could have been the infection demonstrated by the left-deviating leukocytosis.

A diagnosis of metastatic melanoma in the bladder can be made in almost all terminal patients with hematuria and melanuria by means of cystoscopy. However, this type of diagnosis is rarely of any practical importance. The main organs of the genitourinary 
tract that become involved are: kidney (45\%), bladder (18\%) and prostate (3\%). ${ }^{5}$ Hematuria was present in this patient in the terminal stage of the disease and the site of the lesion causing these symptoms was not determined. A necropsy would have been of great diagnostic value for understanding this rapid and fatal evolution. However the patient's family did not authorize this, and it was not performed.

The incidence of and mortality from melanoma are increasing and no effective treatment for the disseminated disease exists. Programs for prevention and early detection of melanoma are therefore warranted. ${ }^{9}$

\section{REFERENCES}

1. Balch CM, Soong S, Murad TM, et al. A multifactorial analysis of melanoma IV. Prognostic factors in 200 melanoma patients with distant metastasis (Stage III). J Clin Oncol 1983;1(2):126-34.

2. Klaase JM, Kroon BBR. Surgery for melanoma metastatic to the gastrointestinal tract. Br J Surg 1990;77:60-1.

3. Mastrangelo MJ, Baker AR, Katz HR. Melanoma cutâneo. In: De Vita JRVT, Hellman S, Rosenberg SA, editors. Principios y práctica de oncologia, 2a ed. Barcelona: Salvat Editores SA; 1988:1279-326.

4. Capizi PJ, DonohueJH. Metastatic melanoma of the gastrointestinal tract: a review of the literature. Comprehensive Therapy 1994;20(1):20-3.

5. Das Gupta T, Brasfield R. Metastatic melanoma. Cancer 1964;17:1323-39.
6. Kew MC. Hepatic tumors and cysts. In: Feldman M, Fordtran JS, Scharschmidt BF, Sleisenger MH, editors. Gastrointestinal and liver disease, $6^{\text {th }}$ ed. Philadelphia: WB Saunders;1998:1364-87.

7. Foster JH, Ensminger WF. Tratamiento de las metástasis hepáticas. In: De Vita JRVT, Hellman S, Rosenberg SA, editors. Principios y práctica de oncologia, $2^{\mathrm{a}}$ ed. Barcelona: Salvat Editores SA; 1988:1975-88.

8. Dahn MS, Lange MP, Kosir MA. Splanchnic metabolism associated with liver metastasis. Intensive Care Med 1995;21:352-5.

9. Brandberg $\mathrm{Y}$, Bolunf $\mathrm{C}$, Michelson $\mathrm{H}$, et al. Perceived susceptibility to the knowledge of malignant melanoma: screening participants versus general population. Prev Med 1996;25:170-7.

\section{publishing information}

Laura Cotta Ornellas, MD. Clinical fellow, a fourth year medical graduate student of Gastroenterology Division, Universidade Federal de São Paulo/ Escola Paulista de Medicina, São Paulo, Brazil.

Valéria Pereira Lanzoni, MD. Professor of Department of Pathology,

Universidade Federal de São Paulo/Escola Paulista de Medicina, São Paulo, Brazil. Carlos Fischer de Toledo, MD. Professor of Gastroenterology Division,

Universidade Federal de São Paulo/Escola Paulista de Medicina, São Paulo, Brazil.

Sources of funding: Not declared

Conflict of interest: Not declared

Last received: 27 April 1999

Accepted: 22 September 1999

Address for correspondence:

Carlos Fischer de Toledo

Departamento de Medicina, Disciplina de Gastroenterologia,

Universidade Federal de São Paulo/Escola Paulista de Medicina

Rua Botucatu, 740 - $2^{\circ}$ andar

São Paulo/SP - Brazil - CEP 04023-900

E-mail: fischer@gastro.epm.br 